recognize owing to surface differences, we are no less right to be cautious of false analogies that latch on to surface similarities between two disparate situations but ignore critical differences between them. There is, I believe, a crucial difference between the principles governing learning or the acquisition of knowledge and those governing evolution by natural selection. Natural selection is blind: all that matters is that, for some reason or other, certain phenotypes are more likely to reproduce themselves than others. Those phenotypes have no knowledge of what they are doing that is so successful. Indeed. Natural selection is not interested in the mechanisms or processes underlying successful adaptations: it selects only for consequences.

For Plotkin, however, behaviour that acts upon and is adapted to the world is knowledge about the world - because it is informed by constraints imposed by the world. One can see what is meant here, but still believe that an important distinction is being lost by this extension of the notion of knowledge. Thus Plotkin wants to argue that, just as when I cough to attract someone's attention, I am not simply coughing as part of normal respiratory function, so a male dog who lifts his back leg to urinate is not just urinating, he is scent marking. So he is. But the difference between my coughing and the dog's urinating is that I know what I am up to and the dog (in this instance) does not. His behaviour is 'instinctive': it is not an intentional action performed in order to achieve a certain outcome he has in mind. But when we turn to the dog's learned changes in behaviour, we are dealing with a quite different situation. Here it is abundantly clear that the reason why the dog's behaviour changes in accordance with the law of effect is precisely because he learns about the consequences of his actions. In a reasonably strict sense, he does know what he is up to. It is precisely this knowledge that is missing from Skinner's version of the law, and that allows to draw the analogy with evolution by natural selection. But the analogy is flawed.

Plotkin allows the possibility that goal-directed learning is subject to a Lamarckian rather than a Darwinian selection process, but I doubt whether this concession actually addresses the way in which the analogy fails. A rat in a Skinner box may indeed initially generate responses at random without their being directed towards a goal: in other words, the generation of variation may indeed be random and Darwinian rather than directed and Lamarckian. The critical question is how the selection process operates. Here, both Darwinian and Lamarckian processes are blind. But learning is not: we learn to perform some actions rather than others precisely because we know what the consequences of those actions will be.

It would be quite wrong to end on such a churlish note. Plotkin has written a book that is a pleasure to read, will instruct a wide audience and will force most readers to think for themselves about the issues he addresses. He has certainly forced me to address his arguments. That 1 happen to disagree with him is much less important. Other readers should take the opportunity to make up their own minds.

Nicholas Mackintosh is in the Department of Experimental Psychology, University of Cambridge, Downing Street, Cambridge CB2 3EB, UK

\section{Models and molecules}

\section{Barbara Baird}

Receptors: Models for Binding, Trafficking and Signalling. By Douglas A. Lauffenburger and Jennifer J. Linderman, Oxford University Press: 1993. Pp. 365. $£ 50, \$ 69.95$.

Physiological communication and responses are mediated by individual cells that sense their environment through surface receptors binding to external ligands. The central importance of binding has been revealed after many years of work by cell and molecular biologists and biochemists. We now know a great deal about the receptor and ligand molecules that combine to initiate growth and differentiation, immunological defence and a variety of other vital processes. Chemical engineers also seek an understanding of complex cellular processes in the hope of finding practical applications. They compare fairly simple mathematical descriptions and models with experiment.

Lauffenburger and Linderman are chemical engineers who are aware of the powerful synergy that can come from collaboration with cell biologists. Writing with enthusiasm but care, they go a long way towards their stated goal of bridging the gap between these two groups of workers. Even those such as biophysical chemists who already consider themselves part of the bridge will have a lot to gain from the book: it provides a broad view of biomolecular modelling from simple monovalent receptor-ligand binding in solution to complicated receptormediated processes resulting in cellular proliferation, adhesion and migration.

After a friendly introduction comes a meaty chapter on the fundamentals of receptor-ligand binding. This chapter will be useful to cell biologists now beginning to evaluate the binding properties of receptor-ligand systems with simple equilibrium and kinetic measurements and data analyses. The authors develop the appropriate equations in a straightforward way, with cautious use of Scratchard and other types of popular plots. Applications are illustrated with examples from previously published work. The authors provide brief descriptions but little critical assessment of experimental methods.

Chapters 3, 5 and 6 deal with the increasingly complex receptor/ligandmediated processes of trafficking through the cell, signal transduction and cell behavioural responses. As throughout the book, these chapters emphasize the development of descriptive and predictive mathematical models that can be tested experimentally. The focus is necessarily on the results obtained from a limited amount of successful modelling. Although only a handful of cell biologists will be interested in the details, it is worth having the broad picture of progress. In Chapter 4 , the authors draw attention to the subtle physical aspects of receptor-ligand binding on cell surfaces, including probability and diffusion and multivalent binding and crosslinking. Researchers would do well to pay heed to these issues, clearly presented here, because they may well be important in the regulation of some cellular responses.

Each chapter and major section begins with a conversational overview of the biology and mathematics and provides the necessary background. Limitations in the presentation are acknowledged. The book builds on selections from a large amount of literature and gives extensive references to original sources. With the aid of this effective approach and a little effort, cell biologists will be able to learn, or at least learn to appreciate, the power of mathematical modelling. Any maths phobia is alleviated by the authors' verbal explanations of how the equations describe the processes and what a set of mathematical results really means in physical or biological terms. The list of nomenclature at the end of each chapter prevents the head from reeling with abstract symbols of forgettable meaning.

The value of this book to chemical engineers is that it exposes them to the wealth of receptor-mediated cellular processes that are increasingly amenable to mathematics. This guided glimpse of biology comes with an organized set of mathematical tools for advancing molecular understanding. Most importantly, the book provides a common language in which cell biologists and chemical engineers, and those in between, can talk to each other. This is a strong contribution to a surging interdisciplinary effort.

Barbara Baird is in the Department of Chemistry, Baker Laboratory, Cornell University, Ithaca, New York 14853-1301, USA. 